\title{
Research Article \\ Second-Order Leader-Following Consensus of Multiagent Systems with Time Delays
}

\author{
Hong Xia, Ting-Zhu Huang, Jin-Liang Shao, and Jun-Yan Yu \\ School of Mathematical Sciences, University of Electronic Science and Technology of China, Chengdu, Sichuan 611731, China \\ Correspondence should be addressed to Hong Xia; xiahong@uestc.edu.cn
}

Received 18 April 2013; Accepted 2 November 2013

Academic Editor: Stefano Lenci

Copyright (C) 2013 Hong Xia et al. This is an open access article distributed under the Creative Commons Attribution License, which permits unrestricted use, distribution, and reproduction in any medium, provided the original work is properly cited.

\begin{abstract}
This paper is concerned with a leader-following consensus problem of second-order multiagent systems with a constant acceleration leader and time-varying delays. At first, a distributed control protocol for every agent to track the leader is proposed; then by utilizing the Lyapunov-Razumikhin function, the convergence analysis under both fixed and switching interconnection topologies is investigated. For the case of fixed topology, a sufficient and necessary condition is obtained, and for the case of switching topology, a sufficient condition is derived under some assumptions. Finally, simulation examples are provided to demonstrate the effectiveness of the theoretical results.
\end{abstract}

\section{Introduction}

Recent years have witnessed dramatic advances concerned with distributed coordination of multiple agents due to rapid developments of computer science and communication technologies. As an important issue of distributed coordination, the consensus problem has attracted more and more attention from researchers in various fields [1-5]. A special case of the issue is known as the leader-following consensus problems and has been investigated from different perspectives. Hong et al. in [6], for instance, designed distributed observers to track an active leader for second-order continuous multiagents systems. Also, Tang et al. studied the leader-following consensus problem via sampled-data control in [7]. Besides, the authors in [8] explored first-order leader-following consensus algorithms under a directed fixed topology with a time-varying leader. Moreover, the results in [8] were furthered extended to the case of actuator saturation and switching topology in [9].

Due to the limited communication capacity, time delays are sometimes unavoidable in multiagent systems. Olfatisaber and Murray first analyzed the consensus problem with fixed undirected topology and time delays by a frequency domain approach in [1]. Based on the reduced-order Lyapunov-Krasovskii function and linear matrix inequalities
(LMIs), Lin and Jia considered the averaged consensus problem with a switching topology and time-varying delays in [10]. In addition, Hu and Lin analyzed the second-order consensus problem for multiple agents with time-varying delays in terms of the Lyapunov-Razumikhin method in [11]. Note that $[1,10,11]$ studied leaderless consensus problem with time delays. For leader-follower networks, Hu and Hong [12] investigated the second-order consensus problems with fixed and switching topologies with time delays, and Tang et al. generalized the results of [12] to the case of nonuniform time delays in [13], while the scenario in the presence of detectable time delays was addressed in [14]. It is worth pointing out that the velocity of the leader in [12-14] is a constant, and the time delays exist only in the transmission of position between neighbors.

In this paper, we consider the second-order leaderfollowing consensus problem for multi-agent systems, with time delays existing in the transmission of both velocity and position. Additionally, the velocity of the leader is not restricted to be a constant but a linear function of time; that is, the acceleration of the leader can be a nonzero constant. Furthermore, we investigate the consensus problem under both fixed and switching directed interconnection topologies by means of the Lyapunov-Razumikhin function and provide the bounds of the allowable time delays. 
The rest of this paper is organized as follows. Section 2 provides some preliminaries and formulates the leaderfollowing consensus problem. With the proposed consensus protocol, Section 3 presents the convergence analysis of the case of fixed topology, and Section 4 deals with the case of switching topology. Then simulation results are presented in Section 5. Finally, the conclusion is drawn in Section 6.

The following notations will be used throughout this paper. Let $I_{n}$ be an $n \times n$ identity matrix, $\mathbf{1}_{n}=[1 \cdots 1]^{T} \in \mathbb{R}^{n}$, $0_{n \times n}$ represents an $n \times n$ zero matrix. Given a matrix, the superscripts " $T$ ", and " -1 " stand for matrix transposition and matrix inverse respectively; $\Lambda(\cdot)$ denotes the set of all eigenvalues of the matrix; $\|\cdot\|$ denotes the spectral norm; $\lambda_{\max }(\cdot)$ and $\lambda_{\text {min }}(\cdot)$ denote its maximum and minimum eigenvalues, respectively. $\operatorname{diag}\left\{b_{1}, \ldots, b_{n}\right\}$ denotes a diagonal matrix with diagonal elements being $b_{1}, \ldots, b_{n}$. Given a complex number $\mu \in \mathbb{C}, \operatorname{Re}(\mu), \operatorname{Im}(\mu)$ and $|\mu|$ are its real part, imaginary part, and modulus, respectively. $\otimes$ denotes the Kronecker product.

\section{Preliminaries and Formulation}

Let $\mathscr{G}=(\mathscr{V}, \mathscr{E}, A)$ be a directed graph with a node set $\mathscr{V}=\{1,2, \ldots, n\}$, an edge set $\mathscr{E} \subseteq \mathscr{V} \times \mathscr{V}$, and a weighted adjacency matrix $A=\left[a_{i j}\right]$ with nonnegative elements $a_{i j}$. Node $i$ represents agent $i$, and an edge in $\mathscr{E}$ is denoted by an ordered pair $(j, i)$, where $(j, i) \in \mathscr{E}$ if and only if that agent $i$ can access the state information of agent $j$. The index set of neighbors of node $i$ is denoted by $\mathcal{N}_{i}=\{j \in \mathscr{V}:(j, i) \in \mathscr{E}\}$. For $i, j \in \mathscr{V}, j \in \mathcal{N}_{i} \Leftrightarrow a_{i j}>0$. Moreover, we assume that $a_{i i}=0$ for all $i \in \mathscr{V}$. The indegree and outdegree of node $i$ are, respectively, defined as $d_{\text {in }}(i)=\sum_{j=1}^{i} a_{i j}, d_{\text {out }}(i)=\sum_{j=1}^{i} a_{j i}$. A digraph is called balanced if $\sum_{j=1}^{i} a_{i j}=\sum_{j=1}^{i} a_{i j}$ for all of its nodes. The Laplacian matrix $L=\left[l_{i j}\right] \in \mathbb{R}^{n \times n}$ associated with $A$ is defined as $l_{i i}=\sum_{j=1, j \neq i}^{n} a_{i j}$ and $l_{i j}=-a_{i j}$. Clearly, $L$ has at least one zero eigenvalue with a corresponding eigenvector $\mathbf{1}_{n}$.

A directed path is a sequence of edges in a directed graph of the form $\left(i_{1}, i_{2}\right),\left(i_{2}, i_{3}\right), \ldots$, where $i_{j} \in \mathscr{V}$. If there exists a path from node $j$ to node $i$, we say that $i$ is reachable from $j$. Moreover, $i$ is to said be globally reachable if there is a path from any other node to it. A directed tree is a directed graph, where there exists a node, called the root, such that any other node of the digraph can be reached by one and only one path starting at the root. A spanning tree of a digraph is a directed tree formed by graph edges that connect all the nodes of the graph.

Consider a multiagent system depicted by a graph $\overline{\mathscr{G}}$, which consists of $n$ followers (related to graph $\mathscr{G}$ ) and one leader (labeled as node 0 ) with directed edges from node 0 to some nodes. The leader adjacency matrix associated with graph $\mathscr{G}$ is defined as a matrix $B=\operatorname{diag}\left\{b_{1}, \ldots, b_{n}\right\}$, where $b_{i}>0$ if node 0 is a neighbor of node $i$ and $b_{i}=0$ otherwise.

The matrix $H=L+B$ plays an important role in the analysis of leader-following consensus problem, the following lemma shows a relationship between the positive stability of $H$ and the connectedness of graph $\overline{\mathscr{G}}$.
Lemma 1 (see [12]). The matrix $H=L+B$ is positive stable if and only if node 0 is globally reachable in $\overline{\mathscr{G}}$.

Remark 2. It is straightforward to verify that $H=L+B$ is positive stable if and only if the opposite graph, which is formed by changing the orientation of each $\operatorname{arc}$ of $\overline{\mathscr{G}}$, has a spanning tree rooted at node 0 .

All the considered $n$ followers in this paper move in a $p$ dimensional space, with the kinematic of each follower being described by a double integrator of the form:

$$
\dot{x}_{i}=v_{i}, \quad \dot{v}_{i}=u_{i},
$$

where $x_{i}, v_{i}, u_{i} \in \mathbb{R}^{p}$ denote the position, velocity, and control input of follower $i$, respectively. The dynamics of the leader is expressed as follows:

$$
\dot{x}_{0}=v_{0}, \quad \dot{v}_{0}=a_{0},
$$

where $a_{0} \in \mathbb{R}^{p}$ is the constant acceleration. The leaderfollowing consensus of system (1)-(2) is said to be achieved if the states of followers satisfy $\lim _{t \rightarrow \infty}\left(x_{i}(t)-x_{0}(t)\right)=0$ and $\lim _{t \rightarrow \infty}\left(v_{i}(t)-v_{0}(t)\right)=0, i=1, \ldots, n$.

In this paper, we are interested in discussing the leaderfollowing consensus problem in multi-agent systems for both fixed and switching topologies, with the transmitted information time delay $\tau(t)$. To solve such a problem, the following neighbored-based protocol is used:

$$
\begin{gathered}
u_{i}(t)=a_{0}+\sum_{j \in \mathcal{N}_{i}} a_{i j}(t)\left[k_{1}\left(x_{j}(t-\tau)-x_{i}(t-\tau)\right)\right. \\
\left.+k_{2}\left(v_{j}(t-\tau)-v_{i}(t-\tau)\right)\right] \\
+b_{i}(t)\left[k_{1}\left(x_{0}(t-\tau)-x_{i}(t-\tau)\right)\right. \\
\left.+k_{2}\left(v_{0}(t-\tau)-v_{i}(t-\tau)\right)\right]
\end{gathered}
$$

where $k_{1}, k_{2}>0$ are control parameters, $a_{0}$ is the constant acceleration of the leader, and the time-varying delay $\tau(t)>$ 0 is a continuously differentiable and bounded function. Note that $u_{i}$ in (3) depends on the position information and velocity information, all with time delay $\tau(t)$, of its neighbors and itself. When each follower can get the velocity feedback without time delay, only from the leader and itself, and when the leader moves at a constant velocity (i.e., $a_{0} \equiv 0$ ), protocol (3) becomes

$$
\begin{aligned}
u_{i}(t)= & \sum_{j \in \mathcal{N}_{i}} a_{i j}(t)\left[k_{1}\left(x_{j}(t-\tau)-x_{i}(t-\tau)\right)\right] \\
& +b_{i}(t)\left[k_{1}\left(x_{0}(t-\tau)-x_{i}(t-\tau)\right)\right] \\
& +k\left(v_{0}(t)-v_{i}(t)\right),
\end{aligned}
$$

which is equivalent to protocol (6) in [12].

To describe the variable topologies, we define a piecewise constant switching function $\sigma(t):[0, \infty] \rightarrow \mathscr{I}_{\Gamma}$, where $\mathscr{I}_{\Gamma} \subset \mathbb{N}$ is the index set associated with the elements of $\Gamma$. The set $\Gamma$ is finite because at most a digraph with $n$ nodes is complete and has $n(n-1)$ edges. 
Let $\bar{x}_{i}=x_{i}-x_{0}, \bar{v}_{i}=x_{i}-v_{0}$; then (3) can be written as

$$
\begin{gathered}
u_{i}(t)=a_{0}+\sum_{j \in \mathcal{N}_{i}} a_{i j}(t)\left[k_{1}\left(\bar{x}_{j}(t-\tau)-\bar{x}_{i}(t-\tau)\right)\right. \\
\left.+k_{2}\left(\bar{v}_{j}(t-\tau)-\bar{v}_{i}(t-\tau)\right)\right] \\
-b_{i}(t)\left[k_{1} \bar{x}_{i}(t-\tau)+k_{2} \bar{v}_{i}(t-\tau)\right] .
\end{gathered}
$$

By taking $\bar{x}=\left(\bar{x}_{1}^{T}, \ldots, \bar{x}_{n}^{T}\right)^{T}, \bar{v}=\left(\bar{v}_{1}^{T}, \ldots, \bar{v}_{n}^{T}\right)^{T}$, the closedloop system (1) with protocol (3) can be expressed in a matrix form:

$$
\begin{gathered}
\dot{\bar{x}}=\bar{v}, \\
\dot{\bar{v}}=-k_{1}\left(L_{\sigma}+B_{\sigma}\right) \otimes I_{p} \bar{x}(t-\tau) \\
-k_{2}\left(L_{\sigma}+B_{\sigma}\right) \otimes I_{p} \bar{v}(t-\tau),
\end{gathered}
$$

where $\otimes$ denotes the Kronecker product and $L_{\sigma}, B_{\sigma}$ are the Laplacian matrix and the leader adjacency matrix associated with $\mathscr{G}_{\sigma}$, respectively.

Before discussing the convergence of system (6), we first introduce the following result on the solutions for general functional differential equations [15]. Let $C\left([-\tau, 0], \mathbb{R}^{n}\right)$ be a Banach space of continuous functions $\varphi:[-\tau, 0] \rightarrow \mathbb{R}^{n}$ with a norm $\|\varphi\|_{\mathcal{C}}=\max _{\theta \in[-\tau, 0]}\|\varphi(\theta)\|$. Consider the following time-delay system:

$$
\begin{gathered}
\dot{x}=f\left(t, x_{t}\right), \quad t>0, \\
x(\theta)=\varphi(\theta), \quad \theta \in[-\tau, 0],
\end{gathered}
$$

where $x_{t}(\theta)=x(t+\theta), \forall \theta \in[-\tau, 0], f: \mathbb{R} \times C\left([-\tau, 0], \mathbb{R}^{n}\right) \rightarrow$ $\mathbb{R}^{n}$ is a continuous function and $f(t, 0)=0, \forall t \in \mathbb{R}$.

Lemma 3 (the Lyapunov-Razumikhin Theorem [15]). Let $\phi_{1}, \phi_{2}$, and $\phi_{3}$ be continuous, nonnegative, nondecreasing functions with $\phi_{1}(s)>0, \phi_{2}(s)>0, \phi_{3}(s)>0$ for $s>0$ and $\phi_{1}(0)=\phi_{2}(0)=0$. If there is a continuous function $V(t, x)$ such that

$$
\phi_{1}(\|x\|) \leq V(t, x) \leq \phi_{2}(\|x\|), \quad t \in \mathbb{R}, x \in \mathbb{R}^{n} .
$$

In addition, there exists a continuous nondecreasing function $\phi(s)$ with $\phi(s)>s, s>0$ such that the derivative of $V$ along the solution $x(t)$ of (7) satisfies

$$
\begin{gathered}
\dot{V}(t, x) \leq-\phi_{3}(\|x\|) \\
\text { if } V(t+\theta, x(t+\theta))<\phi(V(t, x(t))), \quad \theta \in[-\tau, 0] ;
\end{gathered}
$$

then the solution $x=0$ is uniformly asymptotically stable.

The function $V(t, x)$ which satisfies (8) and (9) in Lemma 3 is usually called the Lyapunov-Razumikhin function. sections.

The following lemmas are needed in the subsequent

Lemma 4 (see [16]). For any two real vectors $a$ and $b$ with the same dimension, one has

$$
2 a^{T} b \leq a^{T} \Psi^{-1} a+b^{T} \Psi b,
$$

where $\Psi$ is any positive definite matrix with an appropriate dimension.

Lemma 5 (the Schur Complement Theorem [17]). Let $S$ be a symmetric matrix partitioned into blocks:

$$
S=\left(\begin{array}{ll}
A & B \\
B^{T} & C
\end{array}\right)
$$

where both $A$ and $C$ are square; then the following properties are equivalent:

(1) $S$ is positive definite;

(2) $C$ and $A-B C^{-1} B^{T}$ are positive definite;

(3) $A$ and $C-B^{T} A^{-1} B$ are positive definite.

Give a complex polynomial $R(z)=\rho_{0}+\rho_{1} z+\cdots+\rho_{n} z^{n}$, where $\rho_{i} \in \mathbb{C}, i=0,1, \ldots, n$. Substituting $z=\mathrm{i} \omega$ into $R(z)$, we have $R(\mathrm{i} \omega)=\alpha(\omega)+\mathrm{i} \beta(\omega)$, where $\alpha(\omega), \beta(\omega) \in \mathbb{R}$, and $\mathrm{i}$ is the imaginary unit. The following lemma shows the relationship between the Hurwitz stability of $R(z)$ and the related pair $\alpha(\omega), \beta(\omega)$.

Lemma 6 (the Hermite-Biehler Theorem [18]). The complex polynomial $R(z)$ is Hurwitz stable if and only if the related pair $\alpha(\omega), \beta(\omega)$ is interlaced and $\alpha(0) \beta^{\prime}(0)-\alpha^{\prime}(0) \beta(0)>0$.

\section{Consensus with Fixed Topology}

This section focuses on the convergence analysis of (6) with fixed topology. In this case, the subscript $\sigma$ can be dropped for simplicity. Denote $\varepsilon=\left(\bar{x}^{T}, \bar{v}^{T}\right)^{T}$; we can obtain an error dynamics of (3) as follows:

$$
\dot{\varepsilon}=Y \varepsilon+Z \varepsilon(t-\tau),
$$

where

$$
\begin{array}{r}
Y=\left(\begin{array}{cc}
0_{n \times n} & I_{n} \\
0_{n \times n} & 0_{n \times n}
\end{array}\right) \otimes I_{p}, \quad Z=\left(\begin{array}{cc}
0_{n \times n} & 0_{n \times n} \\
-k_{1} H & -k_{2} H
\end{array}\right) \otimes I_{p}, \\
H=L+B .
\end{array}
$$

By the Newton-Leibnitz formula, we have

$$
\begin{aligned}
\varepsilon(t-\tau) & =\varepsilon(t)-\int_{-\tau}^{0} \dot{\varepsilon}(t+s) d s \\
& =\varepsilon(t)-Y \int_{-\tau}^{0} \varepsilon(t+s) d s-Z \int_{-\tau}^{0} \varepsilon(t-\tau+s) d s
\end{aligned}
$$

Thus, system (12) can be rewritten as

$$
\dot{\varepsilon}=F \varepsilon-Z Y \int_{-\tau}^{0} \varepsilon(t+s) d s-Z^{2} \int_{-\tau}^{0} \varepsilon(t-\tau+s) d s,
$$

where $F=Y+Z$.

The matrix $F$ plays a key role in the convergence analysis of (15); the following lemma shows a sufficient and necessary condition that can guarantee the Hurwitz stability of $F$. 
Lemma 7. The matrix $F$ is Hurwitz stable if and only if $H$ is positive stable and the control parameters satisfy $k_{2}^{2} / k_{1}>$ $\max _{\lambda \in \Lambda(H)}\left(\operatorname{Im}^{2}(\lambda) / \operatorname{Re}(\lambda)|\lambda|^{2}\right)$.

Proof. Let $z$ be an eigenvalue of $F$. Then, one has

$$
\begin{aligned}
\operatorname{det}\left(z I_{2 n p}-F\right) & =\operatorname{det}\left(\left[\begin{array}{cc}
z I_{n} & -I_{n} \\
k_{1} H & z I_{n}+k_{2} H
\end{array}\right] \otimes I_{p}\right) \\
& =\operatorname{det}\left(\left(z\left(z I_{n}+k_{2} H\right)+k_{1} H\right) \otimes I_{p}\right) \\
& =\prod_{i=1}^{n}\left(z^{2}+k_{2} \lambda_{i} z+k_{1} \lambda_{i}\right)^{p},
\end{aligned}
$$

where $\lambda_{i}$ is the $i$ th eigenvalue of $H$. Therefore, the Hurwitz stability of matrix $F$ is equivalent to that of the polynomial: $R(z)=z^{2}+k_{2} \lambda_{i} z+k_{1} \lambda_{i}$. Substituting $z=\mathrm{i} \omega$ into $R(z)$, one gets $R(\mathrm{i} \omega)=-\omega^{2}+k_{2}\left(\operatorname{Re}\left(\lambda_{i}\right)+\mathrm{i} \operatorname{Im}\left(\lambda_{i}\right)\right) \mathrm{i} \omega+k_{1}\left(\operatorname{Re}\left(\lambda_{i}\right)+\right.$ $\left.\operatorname{iIm}\left(\lambda_{i}\right)\right)$. Namely, $\alpha(\omega)=-\omega^{2}-k_{2} \operatorname{Im}\left(\lambda_{i}\right) \omega+k_{1} \operatorname{Re}\left(\lambda_{i}\right)$ and $\beta(\omega)=k_{2} \operatorname{Re}\left(\lambda_{i}\right) \omega+k_{1} \operatorname{Im}\left(\lambda_{i}\right)$. According to Lemma 6, $R(z)$ is Hurwitz stable if and only if the following conditions hold:

(i) $\alpha(\omega)=0$ has two distinct real roots $\alpha_{1}<\alpha_{2}$;

(ii) the interlaced condition holds; that is, $\alpha_{1}<\beta_{1}<\alpha_{2}$, where $\beta_{1}$ is the unique root of $\beta(\omega)=0$;

(iii) $\alpha(0) \beta^{\prime}(0)-\alpha^{\prime}(0) \beta(0)>0$.

Considering condition (i), $\alpha(\omega)=0$ has two distinct real roots if and only if $\Delta_{\alpha}=k_{2}^{2} \operatorname{Im}^{2}\left(\lambda_{i}\right)+4 k_{1} \operatorname{Re}\left(\lambda_{i}\right)>0$. Noting that $\operatorname{Re}\left(\lambda_{i}\right)>0$, since $H$ is positive stable, condition (i) holds obviously. In addition, the roots in condition (ii) can be expressed as $\alpha_{1}=\left(-k_{2} \operatorname{Im}\left(\lambda_{i}\right)-\sqrt{\Delta_{\alpha}}\right) / 2, \alpha_{2}=\left(-k_{2} \operatorname{Im}\left(\lambda_{i}\right)+\right.$ $\left.\sqrt{\Delta_{\alpha}}\right) / 2$ and $\beta_{1}=-k_{1} \operatorname{Im}\left(\lambda_{i}\right) / k_{2} \operatorname{Re}\left(\lambda_{i}\right)$. It is easy to check $\alpha_{1}<\beta_{1}<\alpha_{2} \Leftrightarrow k_{2}^{2} / k_{1}>\operatorname{Im}^{2}(\lambda) / \operatorname{Re}(\lambda)|\lambda|^{2}$. Furthermore, using the fact that $\left|\lambda_{i}\right|>0$, the condition (iii) $\alpha(0) \beta^{\prime}(0)-$ $\alpha^{\prime}(0) \beta(0)=\operatorname{Re}^{2}\left(\lambda_{i}\right) k_{1} k_{2}+k_{1} k_{2} \operatorname{Im}^{2}\left(\lambda_{i}\right)=k_{1} k_{2}\left|\lambda_{i}\right|^{2}>0$ holds.

Theorem 8. For system (12), suppose that the control parameters satisfy

$$
\frac{k_{2}^{2}}{k_{1}}>k_{0}=\max _{\lambda \in \Lambda(H)} \frac{\operatorname{Im}^{2}(\lambda)}{\operatorname{Re}(\lambda)|\lambda|^{2}} .
$$

Then, there exists a constant $\tau_{0}>0$ (which will be estimated in the following (22)) such that when $\tau<\tau_{0}$,

$$
\lim _{t \rightarrow \infty} \varepsilon(t)=0 ;
$$

namely, the leader-following consensus is reached asymptotically if and only if the graph $\overline{\mathscr{G}}$ has a spanning tree.

Proof. Sufficiency. Since the graph $\overline{\mathscr{G}}$ has a spanning tree, by Remark $2, H$ is positive stable. Then, it follows from Lemma 7 that $F$ is Hurwitz stable. Therefore, there exists a positive definite matrix $P \in \mathbb{R}^{2 n p \times 2 n p}$ such that

$$
P F+F^{T} P=-I_{2 n p} .
$$

Take the Lyapunov-Razumikhin function $V(\varepsilon)=\varepsilon^{T} P \varepsilon$. Obviously, $\lambda_{\text {min }}(P)\|\varepsilon\|^{2} \leq V(\varepsilon(t)) \leq \lambda_{\max }(P)\|\varepsilon\|^{2}$. In addition, based on (15), we have

$$
\begin{aligned}
\dot{V}(\varepsilon)= & \varepsilon^{T}\left(P F+F^{T} P\right) \varepsilon-2 \int_{-\tau}^{0} \varepsilon^{T} P Z Y \varepsilon(t+s) d s \\
& -2 \int_{-\tau}^{0} \varepsilon^{T} P Z^{2} \varepsilon(t-\tau+s) d s \\
\leq & \varepsilon^{T}\left(P F+F^{T} P\right) \varepsilon+\tau \varepsilon^{T} P Z Y P^{-1} Y^{T} Z^{T} P \varepsilon \\
& +\int_{-\tau}^{0} \varepsilon^{T}(t-r+s) P \varepsilon(t-\tau+s) d s \\
& +\tau \varepsilon^{T} P Z^{2} P^{-1}\left(Z^{2}\right)^{T} P \varepsilon \\
& +\int_{-\tau}^{0} \varepsilon^{T}(t-\tau+s) P \varepsilon(t-\tau+s) d s,
\end{aligned}
$$

where the inequality is obtained from Lemma 4 by setting $a_{1}=-Y^{T} Z^{T} P \varepsilon, \Psi=P, b_{1}=\varepsilon(t+s), a_{2}=-\left(Z^{2}\right)^{T} P \varepsilon$, $b_{2}=\varepsilon(t-r+s)$. Then, take $\phi(s)=q s$ for some constant $q>1$. In the case of $V(\varepsilon(t+\theta))<q V(\varepsilon(t)),-2 \tau \leq \theta \leq 0$, the time derivative of $V(\varepsilon(t))$ gives

$$
\begin{gathered}
\dot{V}(\varepsilon) \leq-\varepsilon^{T} \varepsilon+\tau \varepsilon^{T}\left(P Z Y P^{-1} Y^{T} Z^{T} P+P Z^{2} P^{-1}\left(Z^{2}\right)^{T} P\right. \\
+2 q P) \varepsilon .
\end{gathered}
$$

Consequently, $\dot{V}(\varepsilon) \leq-\eta \varepsilon^{T} \varepsilon$ for some constant $\eta>0$ if

$$
\tau<\tau_{0}=\frac{1}{\left\|P Z Y P^{-1} Y^{T} Z^{T} P+P Z^{2} P^{-1}\left(Z^{2}\right)^{T} P+2 q P\right\|} .
$$

Hence, system (12) is asymptotically stable, invoking Lemma 3.

Necessity. As a special case of (15), the system $\dot{\varepsilon}=F \varepsilon$ is asymptotical stable, which implies that $F$ is Hurwitz stable. Hence, by Lemma 7, $H$ is positive stable, and then it follows from Remark 2 that the graph $\overline{\mathscr{G}}$ has a spanning tree.

Remark 9. In the special case where $\tau \equiv 0$ and $b_{i}(t)>0$ for $i=1, \ldots, n$, that is, the system is free of time delay and all the followers have full access to the leader, system (3) becomes

$$
\begin{aligned}
u_{i}(t)= & a_{0}+\sum_{j \in \mathcal{N}_{i}} a_{i j}(t)\left[k_{1}\left(x_{j}(t)-x_{i}(t)\right)\right. \\
& \left.+k_{2}\left(v_{j}(t)-v_{i}(t)\right)\right] \\
& +b_{i}\left[k_{1}\left(x_{0}(t)-x_{i}(t)\right)+k_{2}\left(v_{0}(t)-v_{i}(t)\right)\right],
\end{aligned}
$$

which is equivalent to the protocol (9) in [19]. 


\section{Consensus with Switching Topology}

Consider system (6) with switching topology. Denote $\varepsilon=$ $\left(\bar{x}^{T}, \bar{v}^{T}\right)^{T} ;(6)$ can be equivalently expressed as follows:

$$
\dot{\varepsilon}=Y \varepsilon+Z_{\sigma} \varepsilon(t-\tau),
$$

where

$$
\begin{array}{r}
Y=\left(\begin{array}{cc}
0_{n \times n} & I_{n} \\
0_{n \times n} & 0_{n \times n}
\end{array}\right) \otimes I_{p}, \quad Z_{\sigma}=\left(\begin{array}{cc}
0_{n \times n} & 0_{n \times n} \\
-k_{1} H_{\sigma} & -k_{2} H_{\sigma}
\end{array}\right) \otimes I_{p}, \\
H_{\sigma}=L_{\sigma}+B_{\sigma} .
\end{array}
$$

Following the lines of the analysis of the case with fixed topology, we have

$$
\begin{aligned}
\varepsilon(t-\tau) & =\varepsilon(t)-\int_{-\tau}^{0} \dot{\varepsilon}(t+s) d s \\
& =\varepsilon(t)-Y \int_{-\tau}^{0} \varepsilon(t+s) d s-Z_{\sigma} \int_{-\tau}^{0} \varepsilon(t-\tau+s) d s
\end{aligned}
$$

Then, (24) can be written as

$$
\dot{\varepsilon}=F_{\sigma} \varepsilon-Z_{\sigma} Y \int_{-\tau}^{0} \varepsilon(t+s) d s-Z_{\sigma}^{2} \int_{-\tau}^{0} \varepsilon(t-\tau+s) d s,
$$

where $F_{\sigma}=Y+Z_{\sigma}$.

Before moving on, we first recall some conditions under which the matrix $H_{\sigma}+H_{\sigma}^{T}$ is positive definite. In [12], Hu and Hong proved that $H_{\sigma}+H_{\sigma}^{T}$ is positive definite when the graph $\overline{\mathscr{G}}$ is balanced and node 0 is globally reachable, while the authors presented the corresponding improved result in [7].

Lemma 10 (see [7]). Suppose that the weights for edges of $\overline{\mathscr{G}}_{\sigma}$ satisfy the following assumptions.

(A1) $\sum_{j \in \mathcal{N}_{i}} a_{i j} \geq \sum_{j \in \mathcal{N}_{i}} a_{j i}, i \notin \mathscr{I}, i \in \mathscr{V}$,

(A2) $2 b_{i}+\sum_{j \in \mathcal{N}_{i}} a_{i j}>\sum_{j \in \mathcal{N}_{i}} a_{j i}, i \in \mathscr{I}$,

(A3) node 0 is globally reachable in $\overline{\mathscr{G}}_{\sigma}$,

where $\mathscr{I}$ denotes the index set of neighbors of node 0 , that is, $\mathscr{I}=\left\{b_{i}>0, i \in \mathscr{V}\right\}$; then $H_{\sigma}+H_{\sigma}^{T}$ is positive definite.

Note that $\sum_{j \in \mathcal{N}_{i}} a_{i j}$ and $\sum_{j \in \mathcal{N}_{i}} a_{j i}$ are the indegree and outdegree of node $i$ in $\overline{\mathscr{G}}$, respectively. Then, the assumption (A1) in Lemma 10 implies that the in-degree of node $i$ is no less than its out-degree for $i \notin \mathscr{I}$. Clearly, the condition that $\overline{\mathscr{G}}$ is balanced is a special case of assumptions (A1) and (A2). Also, changing the orientation of each arc of the graph, the assumption (A3) is equivalent to a spanning tree (rooted at node 0 ) contained in $\overline{\mathscr{G}}$.

Based on Lemma 10 and the fact that the set $\mathscr{I}_{\Gamma}$ is finite, when the weights for edges of $\overline{\mathscr{G}}_{\sigma}$ satisfy the assumptions of Lemma $10, \mu=\min _{\sigma}\left\{\lambda_{\min }\left(H_{\sigma}+H_{\sigma}^{T}\right)\right\}>0$ is well defined.

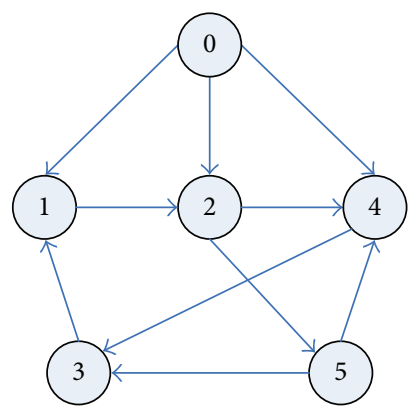

FIGURE 1: Interaction topology $\overline{\mathscr{G}}_{1}$.

Now, we give the main result of leader-following consensus problem with switching topology.

Theorem 11. For system (24), suppose that the control parameters satisfy

$$
k_{2}>k_{1}>k^{*}=\frac{1}{2 \mu}
$$

and the graph $\overline{\mathscr{G}}$ satisfies the assumptions in Lemma 10. Take $\tau<\tau_{1}$ (which will be estimated in the following (34)); then one has

$$
\lim _{t \rightarrow \infty} \varepsilon(t)=0
$$

that is, the leader-following consensus is reached asymptotically.

Proof. Take the Lyapunov-Razumikhin function $V(\varepsilon)=$ $\varepsilon^{T} \Phi \mathcal{\varepsilon}$, where

$$
\Phi=\left(\begin{array}{ll}
k_{2} I_{n} & k_{1} I_{n} \\
k_{1} I_{n} & k_{2} I_{n}
\end{array}\right) \otimes I_{p}
$$

is positive definite for $k_{2}>k_{1}>0$.

Following a similar line to the proof of Theorem 8 , we can obtain

$$
\begin{aligned}
\dot{V}(\varepsilon) \leq & \varepsilon^{T}\left(\Phi F_{\sigma}+F_{\sigma}^{T} \Phi\right) \varepsilon+\tau \varepsilon^{T} \Phi Z_{\sigma} Y \Phi^{-1} Y^{T} Z \sigma^{T} \Psi \varepsilon \\
& +\int_{-\tau}^{0} \varepsilon^{T}(t-r+s) \Psi \varepsilon(t-\tau+s) d s \\
& +\tau \varepsilon^{T} \Phi Z_{\sigma}^{2} \Psi^{-1}\left(Z_{\sigma}^{2}\right)^{T} \Psi \varepsilon \\
& +\int_{-\tau}^{0} \varepsilon^{T}(t-\tau+s) \Psi \varepsilon(t-\tau+s) d s .
\end{aligned}
$$




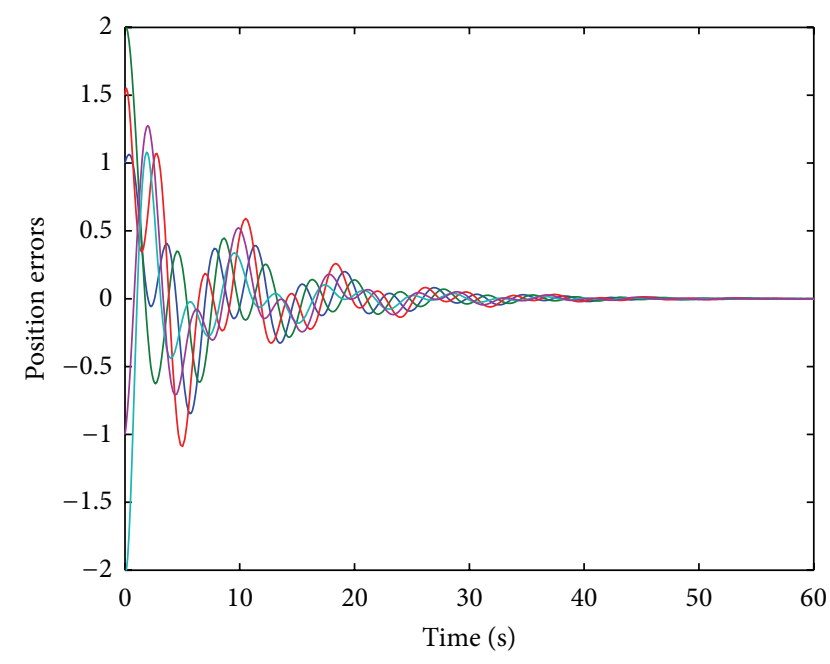

(a)

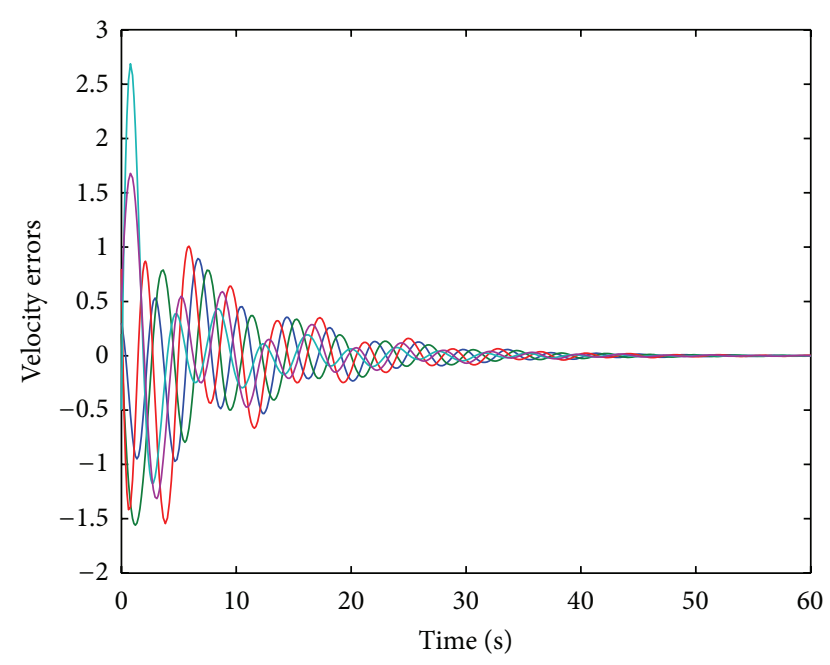

(b)

FIGURE 2: Position errors and velocity errors.

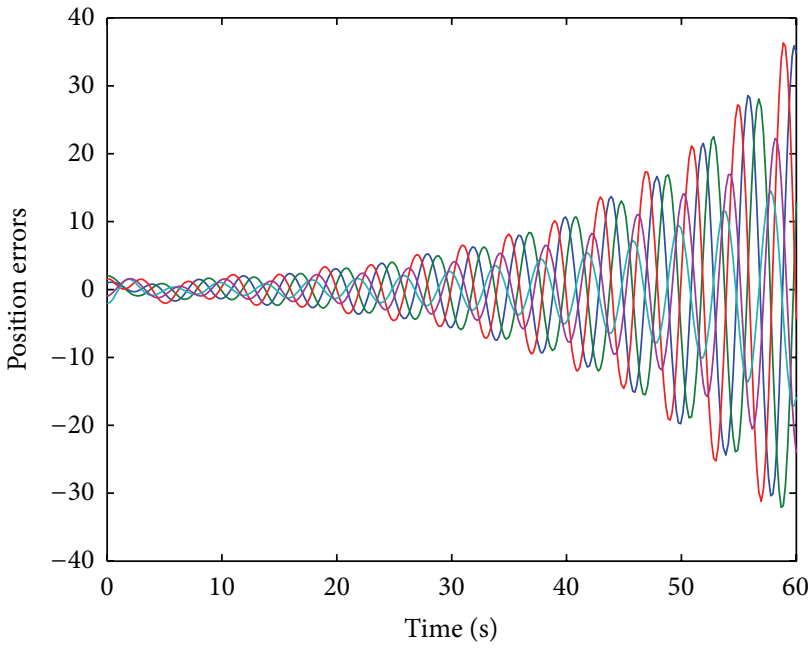

(a)

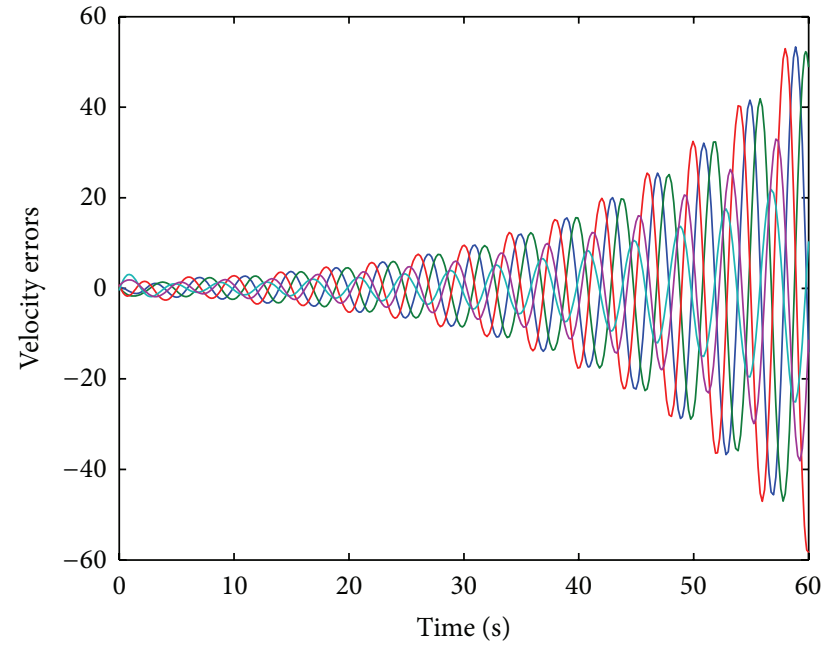

(b)

FIgURe 3: Position errors and velocity errors.

Take $\phi(s)=q s$ for some constant $q>1$. In the case of $V(\varepsilon(t+$ $\theta))<q V(\varepsilon(t)),-2 \tau \leq \theta \leq 0$, we have

$$
\begin{aligned}
\dot{V}(\varepsilon) \leq-\varepsilon^{T} M_{\sigma} \varepsilon+\tau \varepsilon^{T}( & \Psi Z_{\sigma} Y \Phi^{-1} Y^{T} Z_{\sigma}^{T} \Phi \\
& \left.+\Phi Z_{\sigma}^{2} \Phi^{-1}\left(Z_{\sigma}^{2}\right)^{T} \Phi+2 q \Phi\right) \varepsilon
\end{aligned}
$$

where

$$
\begin{aligned}
& M_{\sigma} \\
& =-\left(\Phi F_{\sigma}+F_{\sigma}^{T} \Phi\right) \\
& =\left(\begin{array}{cc}
k_{1}^{2}\left(H_{\sigma}+H_{\sigma}^{T}\right) & k_{1} k_{2}\left(H_{\sigma}+H_{\sigma}^{T}\right)-k_{2} I_{n} \\
k_{1} k_{2}\left(H_{\sigma}+H_{\sigma}^{T}\right)-k_{2} I_{n} & k_{2}^{2}\left(H_{\sigma}+H_{\sigma}^{T}\right)-2 k_{1} I_{n}
\end{array}\right) \otimes I_{p} .
\end{aligned}
$$

From Lemma 5, $M_{\sigma}$ is positive definite if the control parameters satisfy (28). Furthermore, $\dot{V}(\varepsilon)$ is negative definite if

$$
\begin{aligned}
& \tau<\tau_{1} \\
& =\frac{\min _{\sigma}\left\{\lambda_{\min }\left\{M_{\sigma}\right\}\right\}}{\max _{\sigma}\left\|\Phi Z_{\sigma} Y \Phi^{-1} Y^{T} Z_{\sigma}^{T} \Phi+\Phi Z_{\sigma}^{2} \Phi^{-1}\left(Z_{\sigma}^{2}\right)^{T} \Phi+2 q \Phi\right\|} .
\end{aligned}
$$

Therefore, the conclusion follows from Lemma 3.

\section{Simulations}

In this section, to illustrate our theoretical results derived in the above section, we provide two numerical simulations. For 


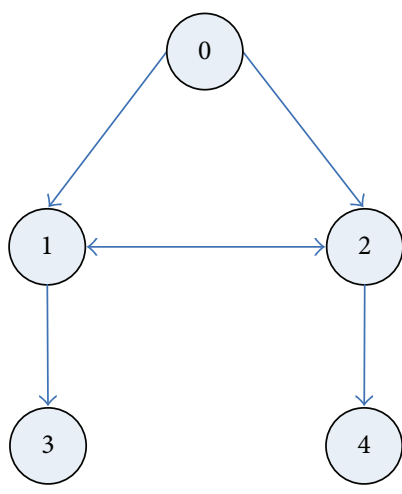

(a)

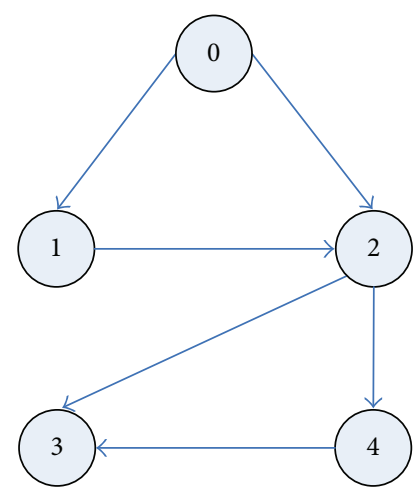

(b)

FIGURE 4: Interconnection topologies $\overline{\mathscr{G}}_{2}$ and $\overline{\mathscr{G}}_{3}$.

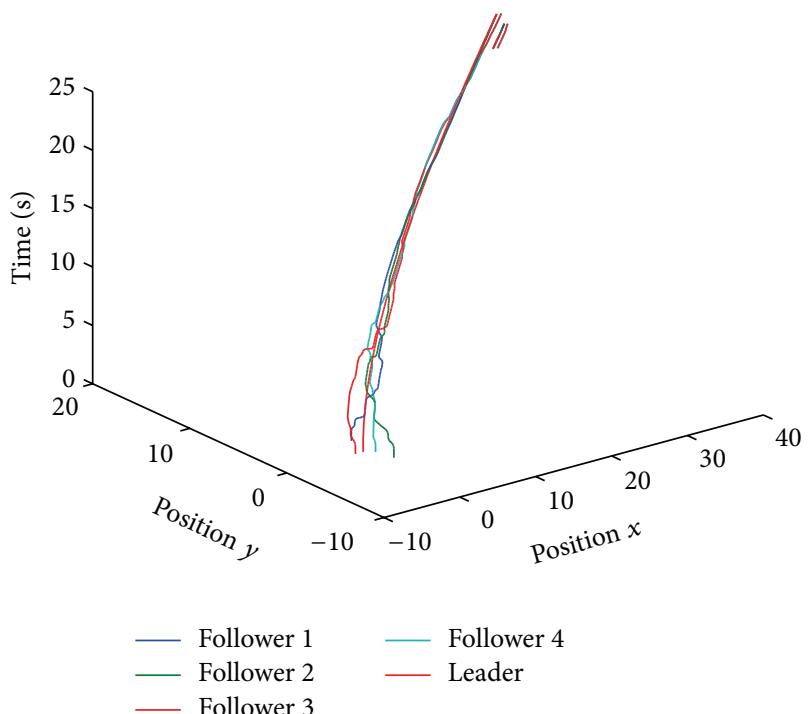

(a)

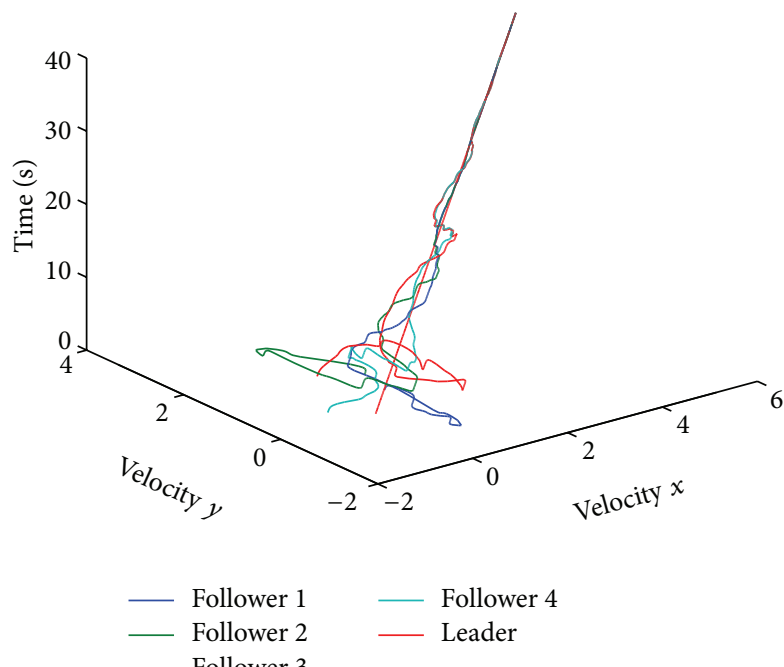

(b)

Figure 5: Position and velocity evolutions.

simplicity, let $a_{i j}=1$ if agent $j$ is a neighbor of agent $i$ and $a_{i j}=0$ otherwise.

Example 12. For the fixed topology case, we consider a multiagent system consisting of one leader and five followers with the interaction graph $\overline{\mathscr{G}}_{1}$ given in Figure 1. It can be noted that $\overline{\mathscr{G}}_{1}$ has a spanning tree. With simple calculations, we can obtain that $k_{0}=0.1295$ and $\tau_{0}=0.0717$. Take $k_{2}=0.5$ and $k_{1}=1$; then $k_{2}^{2} / k_{1}=0.25>0.1295$. Figures $2(\mathrm{a})$ and 2(b) show, respectively, the position errors and velocity errors when $\tau(t)=0.07|\sin t|$. We can see that the five followers can track the leader; Figures 3(a) and 3(b) show, respectively, the position errors and velocity errors when $k_{2}=0.3592$ and $k_{1}=1$, and in this case $k_{2}^{2} / k_{1}=0.129<0.1295$. From Figure 3 , it can be seen that the tracking errors become unbounded.
Example 13. For the switching topology case with $p=2$ (i.e., the agents move in a plane), we consider a multiagent system consisting of one leader and four followers. The interaction topology switches from a set $\left\{\overline{\mathscr{G}}_{2}, \overline{\mathscr{G}}_{3}\right\}$ as shown in Figure 4 . Clearly, the weights of both $\overline{\mathscr{G}}_{2}$ and $\overline{\mathscr{G}}_{3}$ satisfy the assumptions in Lemma 10. With simple calculations, we can obtain that $k^{*}=0.6063$. Take $k_{2}=0.65, k_{1}=0.61$; Figures 5(a) and 5(b) illustrate, respectively, the position evolutions and velocity evolutions with $\tau(t)=0.015|\cos t|$. we can see that the four followers can track the leader.

\section{Conclusion}

In this paper, we consider a second-order consensus problem for a multi-agent system with a constant acceleration 
leader and time-varying delays. Based on the LyapunovRazumikhin function and algebraic theory, we give a sufficient and necessary condition when the interaction topology is fixed and present a sufficient condition when the interaction topology is switching and the weights of graph satisfy some assumptions. Furthermore, two numerical simulations are provided to illustrate the results.

\section{Acknowledgments}

The authors would like to thank the anonymous reviewers and Editor Professor Lenci for their insightful comments and suggestions. This research is supported by NSFC (61104141, 61170309), NSFC Tianyuan foundation (11126104), and Chinese Universities Specialized Research Fund for the Doctoral Program (20110185110020).

\section{References}

[1] R. Olfati-Saber and R. M. Murray, "Consensus problems in networks of agents with switching topology and time-delays," IEEE Transactions on Automatic Control, vol. 49, no. 9, pp. 15201533, 2004.

[2] R. Olfati-Saber, J. A. Fax, and R. M. Murray, "Consensus and cooperation in networked multi-agent systems," Proceedings of the IEEE, vol. 95, no. 1, pp. 215-233, 2007.

[3] R. Olfati-Saber, "Flocking for multi-agent dynamic systems: algorithms and theory," IEEE Transactions on Automatic Control, vol. 51, no. 3, pp. 401-420, 2006.

[4] W. Ren and R. W. Beard, Distributed Consensus in Multi-Vehicle Cooperative Control, Springer, London, UK, 2008.

[5] W. Ren and Y. Cao, Distributed Coordination of Multi-Agent Networks, Springer, London, UK, 2011.

[6] Y. Hong, J. Hu, and L. Gao, "Tracking control for multiagent consensus with an active leader and variable topology," Automatica, vol. 42, no. 7, pp. 1177-1182, 2006.

[7] Z.-J. Tang, T.-Z. Huang, J.-L. Shao, and J.-P. Hu, "Leaderfollowing consensus for multi-agent systems via sampled-data control," IET Control Theory and Applications, vol. 5, no. 14, pp. 1658-1665, 2011.

[8] W. Ren, "Multi-vehicle consensus with a time-varying reference state," Systems \& Control Letters, vol. 56, no. 7-8, pp. 474-483, 2007.

[9] W. Ren, "Consensus tracking under directed interaction topologies: algorithms and experiments," IEEE Transactions on Control Systems Technology, vol. 18, no. 1, pp. 230-237, 2010.

[10] P. Lin and Y. Jia, "Average consensus in networks of multi-agents with both switching topology and coupling time-delay," Physica A, vol. 387, no. 1, pp. 303-313, 2008.

[11] J. Hu and Y. S. Lin, "Consensus control for multi-agent systems with double-integrator dynamics and time delays," IET Control Theory and Applications, vol. 4, no. 1, pp. 109-118, 2010.

[12] J. Hu and Y. Hong, "Leader-following coordination of multiagent systems with coupling time delays," Physica A, vol. 374, no. 2, pp. 853-863, 2007.

[13] Z. Tang, T. Huang, J. Hu, and J. Shao, "Leader-fowing consensus in networks of agents with nonuniform time-varying delays," Mathematical Problems in Engineering, vol. 2012, Article ID 848942, 12 pages, 2012.
[14] W. Zhu and D. Cheng, "Leader-following consensus of secondorder agents with multiple time-varying delays," Automatica, vol. 46, no. 12, pp. 1994-1999, 2010.

[15] J. K. Hale and S. M. Verduyn Lunel, Introduction to FunctionalDifferential Equations, vol. 99, Springer, New York, NY, USA, 1993.

[16] M. Wu, Y. He, and J. She, Stability Analysis and Robust Control of Time-Delay Systems, Science Press, Beijing, China; Springer, Berlin, Germany, 2010.

[17] R. A. Horn and C. R. Johnson, Topics in Matrix Analysis, Cambridge University Press, Cambridge, NY, USA, 1994.

[18] K. Ogata, Discrete-Time Control Systems, Prentice Hall, Englewood Cliffs, NJ, USA, 2nd edition, 1995.

[19] W. Ren, "Second-order consensus algorithm with extensions to switching topologies and reference models," in Proceedings of the American Control Conference (ACC '07), pp. 1431-1436, July 2007. 


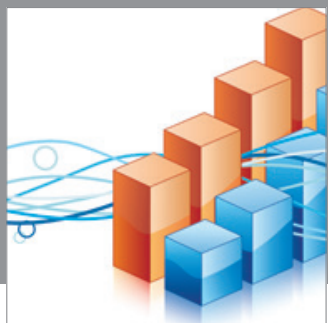

Advances in

Operations Research

mansans

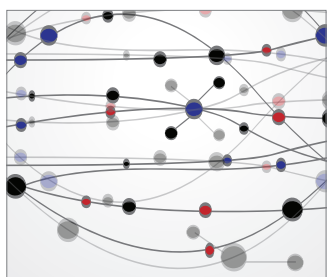

The Scientific World Journal
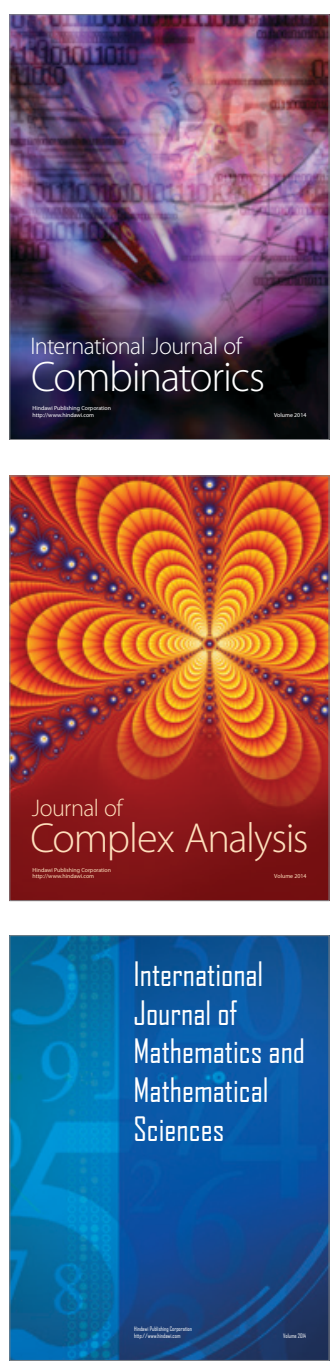
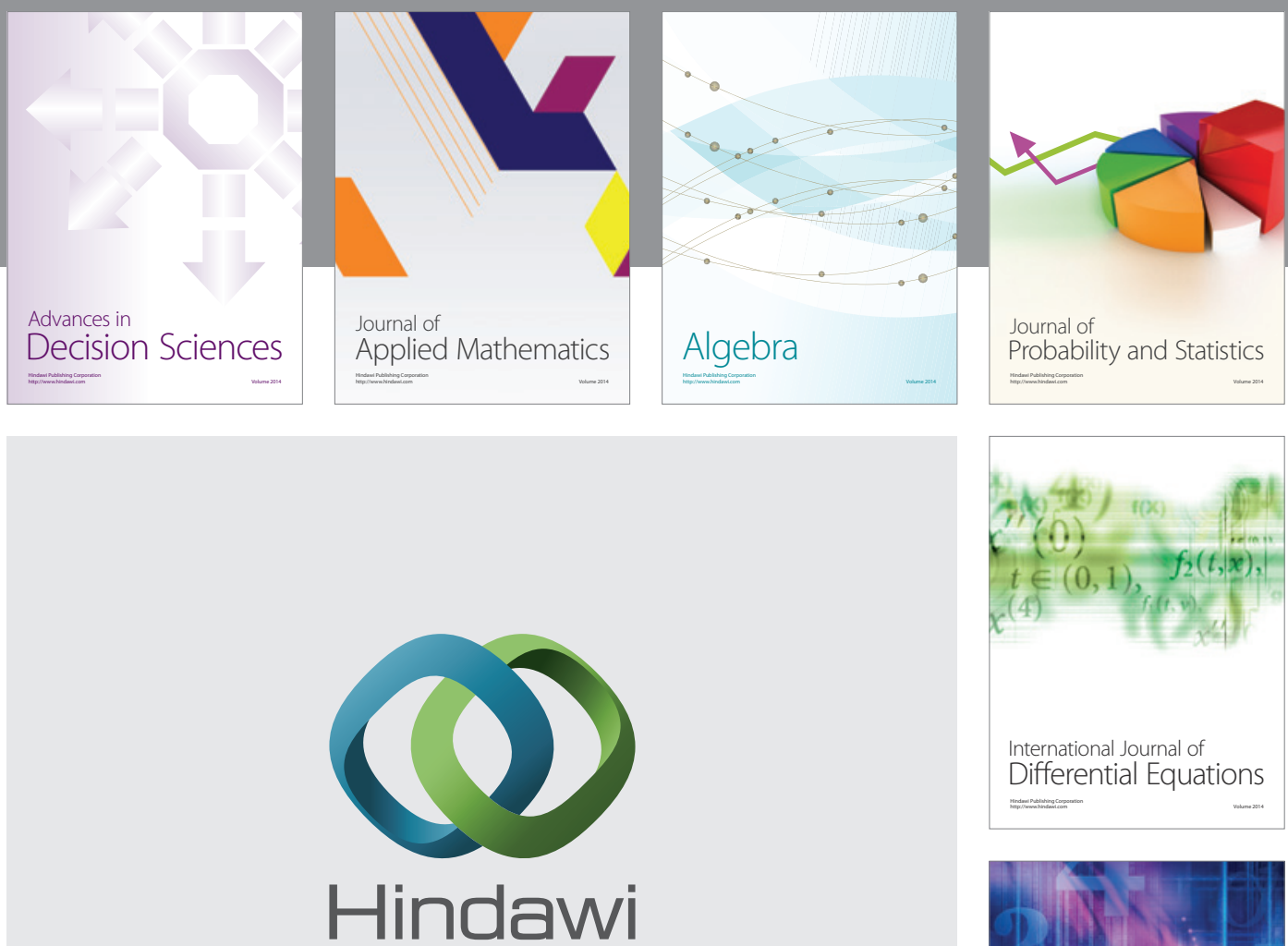

Submit your manuscripts at http://www.hindawi.com
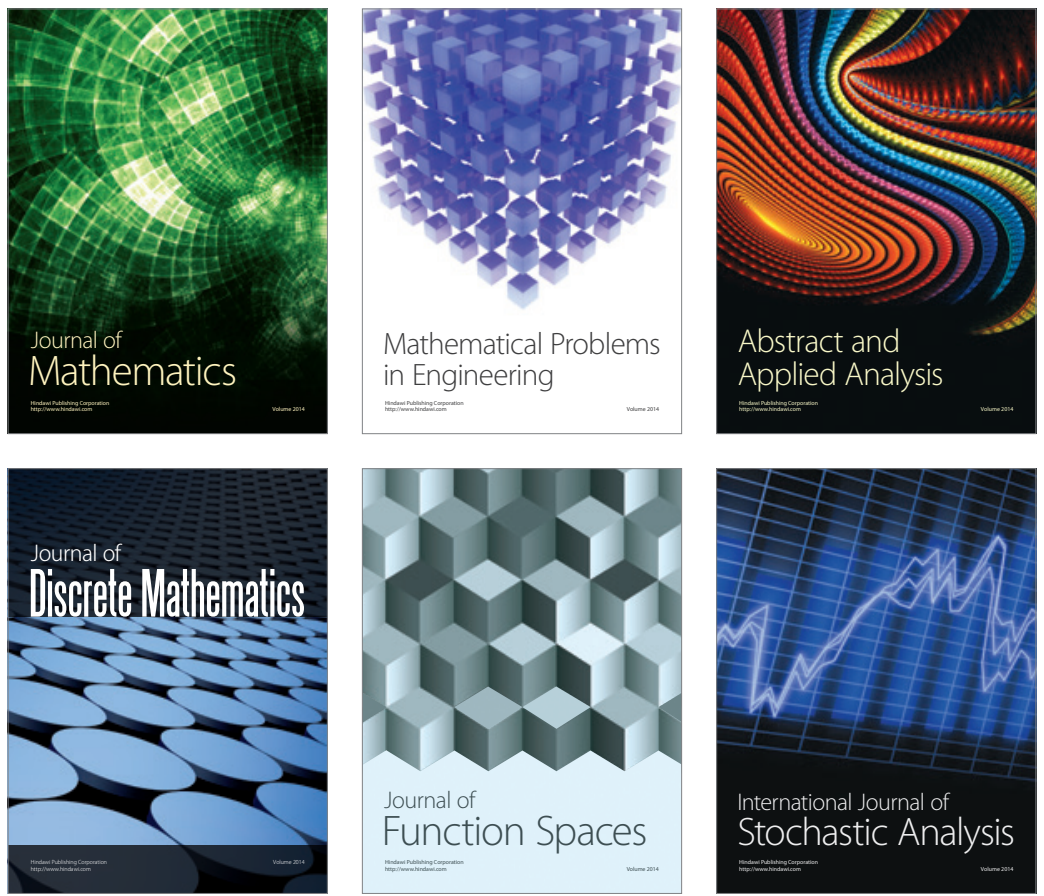

Journal of

Function Spaces

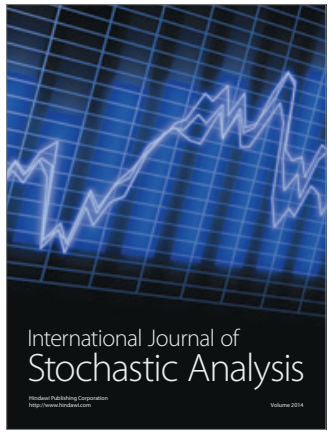

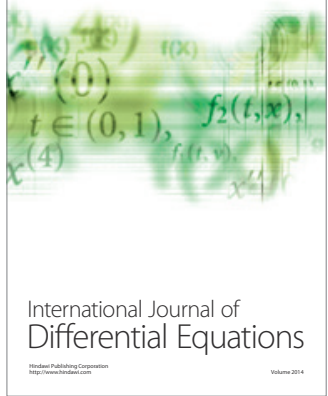
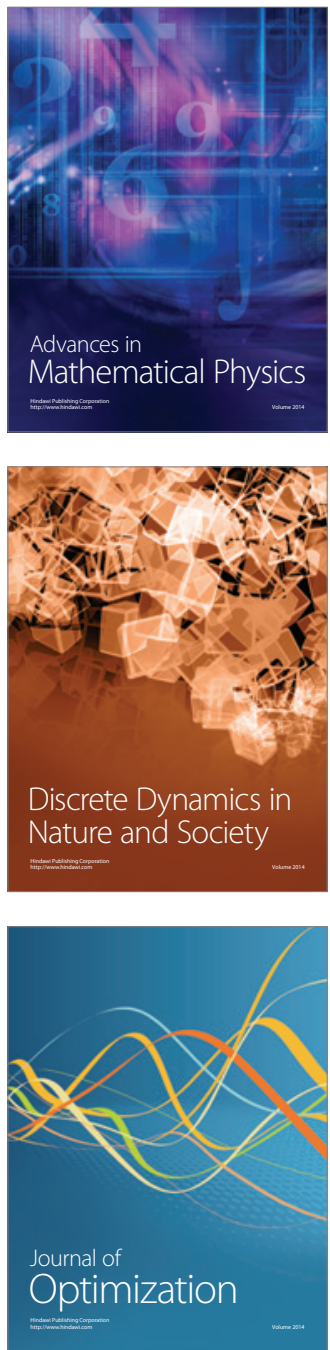